\title{
Galois groups of Mori trinomials and hyperelliptic curves with big monodromy
}

\author{
Yuri G. Zarhin 1
}

Received: 22 December 2014 / Accepted: 12 April 2015 / Published online: 5 May 2015

(C) Springer International Publishing AG 2015

\begin{abstract}
We compute the Galois groups for a certain class of polynomials over the the field of rational numbers that was introduced by Shigefumi Mori and study the monodromy of corresponding hyperelliptic jacobians.
\end{abstract}

Keywords Abelian varieties - Hyperelliptic curves - Tate modules · Galois groups

Mathematics Subject Classification $14 \mathrm{H} 40 \cdot 14 \mathrm{~K} 05 \cdot 11 \mathrm{G} 30 \cdot 11 \mathrm{G} 10$

\section{Mori polynomials, their reductions and Galois groups}

We write $\mathbb{Z}, \mathbb{Q}$ and $\mathbb{C}$ for the ring of integers, the field of rational numbers and the field of complex numbers respectively. If $a$ and $b$ are nonzero integers then we write $(a, b)$ for its (positive) greatest common divisor. If $\ell$ is a prime then $\mathbb{F}_{\ell}, \mathbb{Z}_{\ell}$ and $\mathbb{Q}_{\ell}$ stand for the prime finite field of characteristic $\ell$, the ring of $\ell$-adic integers and the field of $\ell$-adic numbers respectively.

This work was partially supported by a grant from the Simons Foundation (\#246625 to Yuri Zarkhin). This work was started during author's stay at the Max-Planck-Institut für Mathematik (Bonn, Germany) in September of 2013 and finished during the academic year 2013/2014 when the author was Erna and Jakob Michael Visiting Professor in the Department of Mathematics at the Weizmann Institute of Science (Rehovot, Israel): the hospitality and support of both Institutes are gratefully acknowledged.

$凶 \quad$ Yuri G. Zarhin

zarhin@math.psu.edu

1 Department of Mathematics, Pennsylvania State University, University Park, PA 16802, USA 
We consider the subring $\mathbb{Z}[1 / 2] \subset \mathbb{Q}$ generated by $1 / 2$ over $\mathbb{Z}$. We have

$$
\mathbb{Z} \subset \mathbb{Z}\left[\frac{1}{2}\right] \subset \mathbb{Q} .
$$

If $\ell$ is an odd prime then the principal ideal $\ell \mathbb{Z}[1 / 2]$ is maximal in $\mathbb{Z}[1 / 2]$ and

$$
\mathbb{Z}\left[\frac{1}{2}\right] / \ell \mathbb{Z}\left[\frac{1}{2}\right]=\mathbb{Z} / \ell \mathbb{Z}=\mathbb{F}_{\ell} .
$$

If $K$ is a field then we write $\bar{K}$ for its algebraic closure and denote by $\operatorname{Gal}(K)$ its absolute Galois group $\operatorname{Aut}(\bar{K} / K)$. If $u(x) \in K[x]$ is a degree $n$ polynomial with coefficients in $K$ and without multiple roots then we write $\Re_{u} \subset \bar{K}$ for the $n$-element set of its roots, $K\left(\Re_{u}\right)$ the splitting field of $u(x)$ and $\operatorname{Gal}(u / K)=\operatorname{Gal}\left(K\left(\Re_{u}\right) / K\right)$ the Galois group of $u(x)$ viewed as a certain subgroup of the group Perm $\left(\Re_{u}\right) \cong \mathbf{S}_{n}$ of permutations of $\Re_{u}$. As usual, we write $\mathbf{A}_{n}$ for the alternating group, which is the only index 2 subgroup in the full symmetric group $\mathbf{S}_{n}$.

1.1 Discriminants and alternating groups We write $\Delta(u)$ for the discriminant of $u$. We have

$$
0 \neq \Delta(u) \in K, \quad \sqrt{\Delta(u)} \in K\left(\Re_{u}\right) .
$$

It is well known that

$$
\operatorname{Gal}\left(K\left(\Re_{u}\right) / K(\sqrt{\Delta(u)})\right)=\operatorname{Gal}\left(K\left(\Re_{u}\right) / K\right) \cap \mathbf{A}_{n} \subset \mathbf{A}_{n} \subset \mathbf{S}_{n}=\operatorname{Perm}\left(\Re_{u}\right) .
$$

In particular, the permutation (sub)group $\operatorname{Gal}\left(K\left(\Re_{u}\right) / K(\sqrt{\Delta(u)})\right)$ does not contain transpositions; $\Delta(u)$ is a square in $K$ if and only if $\operatorname{Gal}(u / K)$ lies in the alternating (sub)group $\mathbf{A}_{n} \subset \mathbf{S}_{n}$. On the other hand, if $\operatorname{Gal}(u / K)=\mathbf{S}_{n}$ then $\operatorname{Gal}\left(K\left(\Re_{u}\right) / K(\sqrt{\Delta(u)})\right)=\mathbf{A}_{n}$.

If $n$ is odd and char $(K) \neq 2$ then we write $C_{u}$ for the genus $(n-1) / 2$ hyperelliptic curve

$$
C_{u}: y^{2}=u(x)
$$

and $J\left(C_{u}\right)$ for its jacobian, which is an $(n-1) / 2$-dimensional abelian variety over $K$. We write End $\left(J\left(C_{u}\right)\right)$ for the ring of all $\bar{K}$-endomorphisms of $J\left(C_{u}\right)$ and $\operatorname{End}_{K}\left(J\left(C_{u}\right)\right)$ for the (sub)ring of all its $K$-endomorphisms. We have

$$
\mathbb{Z} \subset \operatorname{End}_{K}\left(J\left(C_{u}\right)\right) \subset \operatorname{End}\left(J\left(C_{u}\right)\right)
$$

About 40 years ago Shigefumi Mori [8, Proposition 3, p. 107] observed that if $n=2 g+$ 1 is odd and $\operatorname{Gal}(f / K)$ is a doubly transitive permutation group then $\operatorname{End}_{K}\left(J\left(C_{u}\right)\right)=$ $\mathbb{Z}$. He constructed [8, Theorem 1, p. 105] explicit examples (in all dimensions $g$ ) of 
polynomials (actually, trinomials) $f(x)$ over $\mathbb{Q}$ such that $\operatorname{Gal}(f / \mathbb{Q})$ is doubly transitive and $\operatorname{End}\left(J\left(C_{f}\right)\right)=\mathbb{Z}$.

On the other hand, about 15 years ago the following assertion was proven by the author [17].

Theorem 1.2 Suppose that $\operatorname{char}(K)=0$ and $\operatorname{Gal}(u / K)=\mathbf{S}_{n}$. Then $\operatorname{End}\left(J\left(C_{u}\right)\right)=$ $\mathbb{Z}$.

The aim of this note is to prove that in Mori's examples $\operatorname{Gal}(f / \mathbb{Q})=\mathbf{S}_{2 g+1}$. This gives another proof of the theorem of Mori [8, Theorem 1, p. 105]. Actually, we extend the class of Mori trinomials with $\operatorname{End}\left(J\left(C_{f}\right)\right)=\mathbb{Z}$, by dropping one of the congruence conditions imposed by Mori on the coefficients of $f(x)$. We also prove that the images of $\mathrm{Gal}(\mathbb{Q})$ in the automorphism groups of Tate modules of $J\left(C_{f}\right)$ are almost as large as possible.

1.3 Mori trinomials Throughout this paper, $g, p, b, c$ are integers that enjoy the following properties [8]:

(i) The number $g$ is a positive integer and $p$ is an odd prime. In addition, there is a positive integer $N$ such that $(p-1)^{N} / 2^{N}$ is divisible by $g$. This means that every prime divisor of $g$ is also a divisor of $(p-1) / 2$. This implies that $(p, g)=(p, 2 g)=1$. It follows that if $g$ is even then $p$ is congruent to 1 modulo 4.

(ii) The residue $b \bmod p$ is a primitive root of $\mathbb{F}_{p}=\mathbb{Z} / p \mathbb{Z}$; in particular, $(b, p)=1$.

(iii) The integer $c$ is odd and $(b, c)=(b, 2 g+1)=(c, g)=1$. This implies that $(c, 2 g)=1$.

Mori [8] introduced and studied the monic degree $2 g+1$ polynomial

$$
f(x)=f_{g, p, b, c}(x)=x^{2 g+1}-b x-\frac{p c}{4} \in \mathbb{Z}\left[\frac{1}{2}\right][x] \subset \mathbb{Q}[x],
$$

which we call a Mori trinomial. He proved the following results [8, pp. 106-107].

Theorem 1.4 (Theorem of Mori) Let $f(x)=f_{g, p, b, c}(x)$ be a Mori trinomial. Then:

(i) The polynomial $f(x)$ is irreducible over $\mathbb{Q}_{2}$ and therefore over $\mathbb{Q}$.

(ii) The polynomial $f(x) \bmod p \in \mathbb{F}_{p}[x]$ is a product $x\left(x^{2 g}-b\right)$ of a linear factor $x$ and an irreducible (over $\mathbb{F}_{p}$ ) degree $2 g$ polynomial $x^{2 g}-b$.

(iii) Let $\mathrm{Gal}(f)$ be the Galois group of $f(x)$ over $\mathbb{Q}$ considered canonically as a (transitive) subgroup of the full symmetric group $\mathbf{S}_{2 g+1}$. Then $\mathrm{Gal}(f)$ is a doubly transitive permutation group. More precisely, the transitive $\mathrm{Gal}(f)$ contains a permutation $\sigma$ that is a cycle of length $2 g$.

(iv) For each odd prime $\ell$ every root of the polynomial $f(x) \bmod \ell \in \mathbb{F}_{\ell}[x]$ is either simple or double.

(v) Let us consider the genus g hyperelliptic curve

$$
C_{f}: y^{2}=f(x)
$$

and its jacobian $J\left(C_{f}\right)$, which is a $g$-dimensional abelian variety over $\mathbb{Q}$. Assume additionally that $c$ is congruent to $-p$ modulo 4 . Then $C_{f}$ is a stable curve 
over $\mathbb{Z}$ and $J\left(C_{f}\right)$ has everywhere semistable reduction over $\mathbb{Z}$. In addition, $\operatorname{End}\left(J\left(C_{f}\right)\right)=\mathbb{Z}$.

Remark 1.5 (I) The 2-adic Newton polygon of Mori trinomial $f(x)$ consists of one segment that connects $(0,-2)$ and $(2 g+1,0)$, which are its only integer points. Now the irreducibility of $f(x)$ follows from Eisenstein-Dumas Criterion [9, Corollary 3.6, p. 316], [4, p. 502]. It also follows that the field extension $\mathbb{Q}\left(\Re_{f}\right) / \mathbb{Q}$ is ramified at 2.

(II) If $g=1$ then $2 g+1=3$ and the only doubly transitive subgroup of $\mathbf{S}_{3}$ is $\mathbf{S}_{3}$ itself. Concerning the double transitivity of the Galois group of trinomials of arbitrary degree, see [2, Theorem 4.2, p. 9 and Note 2, p. 10].

(III) The additional congruence condition in Theorem 1.4(v) guarantees that $C_{f}$ has stable (even good) reduction at 2 [8, p. 106]. Mori's proof of the last assertion of Theorem 1.4(v) is based on results of [12] and the equality $\operatorname{End}_{\mathbb{Q}}\left(J\left(C_{f}\right)\right)=\mathbb{Z}$; the latter follows from the double transitivity of Galois groups of Mori trinomials.

Remark 1.6 Since a cycle of even length $2 g$ is an odd permutation, it follows from Theorem 1.4 (iii) that $\mathrm{Gal}(f)$ is not contained in $\mathbf{A}_{2 g+1}$. In other words, $\Delta(f)$ is not a square in $\mathbb{Q}$.

Our first main result is the following statement.

Theorem 1.7 Let $f(x)=f_{g, p, b, c}(x)$ be a Mori trinomial.

(i) If $\ell$ is an odd prime then the polynomial $f(x) \bmod \ell \in \mathbb{F}_{\ell}[x]$ has, at most, one double root and this root (if exists) lies in $\mathbb{F}_{\ell}$.

(ii) There exists an odd prime $\ell \neq p$ such that $f(x) \bmod \ell \in \mathbb{F}_{\ell}[x]$ has a double root $\bar{\alpha} \in \mathbb{F}_{\ell}$. All other roots of $f(x) \bmod \ell$ (in an algebraic closure of $\mathbb{F}_{\ell}$ ) are simple.

(iii) The Galois group $\operatorname{Gal}(f)$ of $f(x)$ over $\mathbb{Q}$ coincides with the full symmetric group $\mathbf{S}_{2 g+1}$. The Galois (sub)group $\operatorname{Gal}\left(\mathbb{Q}\left(\Re_{f}\right) / \mathbb{Q}(\sqrt{\Delta(f)})\right)$ coincides with the alternating group $\mathbf{A}_{2 g+1}$.

(iii') The Galois extension $\mathbb{Q}\left(\Re_{f}\right) / \mathbb{Q}(\sqrt{\Delta(f)})$ is ramified at all prime divisors of 2 . It is unramified at all prime divisors of every odd prime $\ell$.

(iv) Suppose that $g>1$. Then $\operatorname{End}\left(J\left(C_{f}\right)\right)=\mathbb{Z}$.

Remark 1.8 Theorem 1.7 (iv) was proven by Mori under an additional assumption that $c$ is congruent to $-p$ modulo 4 , see Theorem 1.4(v) above.

Remark 1.9 Thanks to Theorem 1.2, Theorem 1.7 (iv) follows readily from Theorem 1.7 (iii).

Remark 1.10 Let $g>1$ and suppose we know that $\mathrm{Gal}(f)$ contains a transposition. Now the double transitivity implies that $\operatorname{Gal}(f)$ coincides with $\mathbf{S}_{2 g+1}$, see [15, Lemma 4.4.3, p. 40].

Let $K$ be a field of characteristic zero and $u(x) \in K[x]$ be a degree $2 g+1$ polynomial without multiple roots. Then the jacobian $J\left(C_{u}\right)$ is a $g$-dimensional abelian 
variety over $K$. For every prime $\ell$ let $T_{\ell}\left(J\left(C_{u}\right)\right)$ be the $\ell$-adic Tate module of $J\left(C_{u}\right)$, which is a free $\mathbb{Z}_{\ell}$-module of rank $2 g$ provided with the canonical continuous action

$$
\rho_{\ell, u}: \operatorname{Gal}(K) \rightarrow \operatorname{Aut}_{\mathbb{Z}_{\ell}}\left(T_{\ell}\left(J\left(C_{u}\right)\right)\right)
$$

of $\operatorname{Gal}(K)[10,14,20]$. There is a Riemann form

$$
e_{\ell}: T_{\ell}\left(J\left(C_{u}\right)\right) \times T_{\ell}\left(J\left(C_{u}\right)\right) \rightarrow \mathbb{Z}_{\ell}
$$

that corresponds to the canonical principal polarization on $J\left(C_{u}\right)$ ([10, Section 20], $\left[21\right.$, Section 1]) and is a nondegenerate (even perfect) alternating $\mathbb{Z}_{\ell}$-bilinear form that satisfies

$$
e_{\ell}(\sigma(x), \sigma(y))=\chi_{\ell}(\sigma) e_{\ell}(\sigma(x), \sigma(y)) .
$$

This implies that the image

$$
\rho_{\ell, u}(\operatorname{Gal}(K)) \subset \operatorname{Aut}_{\mathbb{Z}_{\ell}}\left(T_{\ell}\left(J\left(C_{u}\right)\right)\right)
$$

lies in the (sub)group

$$
\operatorname{Gp}\left(T_{\ell}\left(J\left(C_{u}\right)\right), e_{\ell}\right) \subset \operatorname{Aut}_{\mathbb{Z}_{\ell}}\left(T_{\ell}\left(J\left(C_{u}\right)\right)\right)
$$

of symplectic similitudes of $e_{\ell}[18,19,21]$.

Using results of Chris Hall [5] and the author [21], we deduce from Theorem 1.7 the following statement. (Compare it with [18, Theorem 2.5] and [19, Theorem 8.3].)

Theorem 1.11 Let $K=\mathbb{Q}$ and $f(x)=f_{g, p, b, c}(x) \in \mathbb{Q}[x]$ be a Mori trinomial. Suppose that $g>1$. Then:

(i) For all primes $\ell$ the image $\rho_{\ell, f}(\mathrm{Gal}(\mathbb{Q}))$ is an open subgroup of finite index in $\operatorname{Gp}\left(T_{\ell}\left(J\left(C_{f}\right)\right), e_{\ell}\right)$.

(ii) Let $L$ be a number field and $\mathrm{Gal}(L)$ be its absolute Galois group, which we view as an open subgroup of finite index in $\mathrm{Gal}(\mathbb{Q})$. Then for all but finitely many primes $\ell$ the image $\rho_{\ell, f}(\mathrm{Gal}(L))$ coincides with $\mathrm{Gp}\left(T_{\ell}\left(J\left(C_{f}\right)\right), e_{\ell}\right)$.

The paper is organized as follows. In Sect. 2 we deduce Theorem 1.11 from Theorem 1.7. In Sect. 3 we discuss a certain class of trinomials that is related to Mori polynomials. Section 4 deals with discriminants of Mori polynomials. We prove Theorem 1.7 in Sect. 5.

\section{Monodromy of hyperelliptic jacobians}

Proof of Theorem 1.11 (modulo Theorem 1.7) By Theorem 1.7 (iii), Gal $(f / \mathbb{Q})$ coincides with the full symmetric group $\mathbf{S}_{2 g+1}$. By Theorem 1.7 (iv), End $\left(J\left(C_{f}\right)\right)=\mathbb{Z}$. It follows from Theorem 1.7 (i) that there is an odd prime $\ell$ such that $J\left(C_{f}\right)$ has at $\ell$ a semistable reduction with toric dimension 1 [5]. Now the assertion (i) follows from [21, Theorem 4.3]. The assertion (ii) follows from [5, Theorem 1]. 


\section{Reduction of certain trinomials}

In order to prove Theorem 1.7 (i), we will use the following elementary statement that was inspired by [15, Remark 2, p.42] and [8, p. 106].

Lemma 3.1 (key lemma) Let

$$
u(x)=u_{n, B, C}(x)=x^{n}+B x+C \in \mathbb{Z}[x]
$$

be a monic polynomial of degree $n>1$ such that $B \neq 0$ and $C \neq 0$.

(I) If $u(x)$ has a multiple root then $n$ divides $B$ and $n-1$ divides $C$.

(II) Let $\ell$ be a prime that enjoys the following properties:

(i) $(B, C)$ is not divisible by $\ell$,

(ii) $(n, B)$ is not divisible by $\ell$,

(iii) $(n-1, C)$ is not divisible by $\ell$.

Suppose that $u(x)$ has no multiple roots. Let us consider the polynomial

$$
\bar{u}(x)=u(x) \bmod \ell \in \mathbb{F}_{\ell}[x] .
$$

Then:

(a) $\bar{u}(x)$ has, at most, one multiple root in an algebraic closure of $\mathbb{F}_{\ell}$.

(b) If such a multiple root say, $\gamma$, does exist, then $\ell$ does not divide $n(n-1) B C$ and $\gamma$ is a double root of $\bar{u}(x)$. In addition, $\gamma$ is a nonzero element of $\mathbb{F}_{\ell}$.

(c) If such a multiple root does exist then either the field extension $\mathbb{Q}\left(\Re_{u}\right) / \mathbb{Q}$ is unramified at $\ell$ or a corresponding inertia subgroup at $\ell$ in

$$
\operatorname{Gal}\left(\mathbb{Q}\left(\Re_{u}\right) / \mathbb{Q}\right)=\operatorname{Gal}(u / \mathbb{Q}) \subset \operatorname{Perm}\left(\Re_{u}\right)
$$

is generated by a transposition. In both cases the Galois extension $\mathbb{Q}\left(\Re_{u}\right) /$ $\mathbb{Q}(\sqrt{\Delta(u)})$ is unramified at all prime divisors of $\ell$.

Remark 3.2 The discriminant $\operatorname{Discr}(n, B, C)=\Delta\left(u_{n, B, C}\right)$ of $u_{n, B, C}(x)$ is given by the formula [3, Example 834]

$$
\operatorname{Discr}(n, B, C)=(-1)^{n(n-1) / 2} n^{n} C^{n-1}+(-1)^{(n-1)(n-2) / 2}(n-1)^{n-1} B^{n} .
$$

Remark 3.3 In the notation of Lemma 3.1, assume that $\bar{u}(x)$ has no multiple roots, i.e., $\Delta(u)$ is not divisible by $\ell$. Then obviously $\mathbb{Q}\left(\Re_{u}\right) / \mathbb{Q}$ is unramified at $\ell$. This implies that $\mathbb{Q}\left(\Re_{u}\right) / \mathbb{Q}(\sqrt{\Delta(u)})$ is unramified at all prime divisors of $\ell$.

Proof of Lemma 3.1 (I) Since $u(x)$ has a multiple root, its discriminant

$$
\Delta(u)=(-1)^{n(n-1) / 2} n^{n} C^{n-1}+(-1)^{(n-1)(n-2) / 2}(n-1)^{n-1} B^{n}=0 .
$$

This implies that

$$
n^{n} C^{n-1}= \pm(n-1)^{n-1} B^{n}
$$


Since $n$ and $n-1$ are relatively prime, $n^{n} \mid B^{n}$ and $(n-1)^{n-1} \mid C^{n-1}$. This implies that $n \mid B$ and $(n-1) \mid C$.

(II) We have

$$
\bar{u}(x)=x^{n}+\bar{B} x+\bar{C} \in \mathbb{F}_{\ell}[x]
$$

where

$$
\bar{B}=B \bmod \ell \in \mathbb{F}_{\ell}, \quad \bar{C}=C \bmod \ell \in \mathbb{F}_{\ell} .
$$

The condition (i) implies that either $\bar{B} \neq 0$ or $\bar{C} \neq 0$. The condition (ii) implies that if $\bar{B}=0$ then $n \neq 0$ in $\mathbb{F}_{\ell}$. The condition (iii) implies that if $n-1=0$ in $\mathbb{F}_{\ell}$ then $\bar{C} \neq 0$ and $n \neq 0$ in $\mathbb{F}_{\ell}$. We have

$$
\Delta(\bar{u})=(-1)^{n(n-1) / 2} n^{n} \bar{C}^{n-1}+(-1)^{(n-1)(n-2) / 2}(n-1)^{n-1} \bar{B}^{n}=0
$$

and therefore

$$
n^{n} \bar{C}^{n-1}= \pm(n-1)^{n-1} \bar{B}^{n} .
$$

This implies that if $n-1=0$ in $\mathbb{F}_{\ell}$ then $\bar{C}=0$, which is not the case. This proves that $n-1 \neq 0$ in $\mathbb{F}_{\ell}$. On the other hand, if $\bar{B}=0$ then $\bar{C} \neq 0$ and $n \neq 0$ in $\mathbb{F}_{\ell}$. Then (1) implies that $\bar{C}=0$ and we get a contradiction that proves that $\bar{B} \neq 0$. If $n=0$ in $\mathbb{F}_{\ell}$ then $n-1 \neq 0$ in $\mathbb{F}_{\ell}$ and (1) implies that $\bar{B}=0$, which is not the case. The obtained contradiction proves that $n \neq 0$ in $\mathbb{F}_{\ell}$. If $\bar{C}=0$ then (1) implies that $\bar{B}=0$, which is not the case. This proves that $\ell$ does not divide $n(n-1) B C$.

The derivative of $\bar{u}(x)$ is $\bar{u}^{\prime}(x)=n x^{n-1}+\bar{B}$. We have

$$
x \cdot \bar{u}^{\prime}(x)-n \cdot \bar{u}(x)=-(n-1) \bar{B} x-n \bar{C} .
$$

Suppose $\bar{u}(x)$ has a multiple root $\gamma$ in an algebraic closure of $\mathbb{F}_{\ell}$. Then

$$
\bar{u}(\gamma)=0, \quad \bar{u}^{\prime}(\gamma)=0, \quad n \cdot \gamma \cdot \bar{u}^{\prime}(\gamma)-n \cdot \bar{u}(\gamma)=0 .
$$

Using (2), we conclude that

$$
0=\gamma \cdot \bar{u}^{\prime}(\gamma)-n \cdot \bar{u}(\gamma)=-(n-1) \bar{B} \gamma-n \bar{C}, \quad \gamma=-\frac{n \bar{C}}{(n-1) \bar{B}} \in \mathbb{F}_{\ell} .
$$

This implies that $\gamma \neq 0$.

Notice that the second derivative $\bar{u}^{\prime \prime}(x)=n(n-1) x^{n-2}$. This implies that

$$
\bar{u}^{\prime \prime}(\gamma)=n(n-1) \gamma^{n-2} \neq 0
$$

It follows that $\gamma$ is a double root of $\bar{u}(x)$. This ends the proof of (a) and (b). 
In order to prove (c), notice that there exists a monic degree $n-2$ polynomial $\bar{h}(x) \in \mathbb{F}_{\ell}[x]$ such that

$$
\bar{u}(x)=(x-\gamma)^{2} \cdot \bar{h}(x)
$$

Clearly, $\gamma$ is not a root of $\bar{h}(x)$ and therefore $\bar{h}(x)$ has no multiple roots and is relatively prime to $(x-\gamma)^{2} .^{1}$ By Hensel's Lemma, there exist monic polynomials

$$
h(x), v(x) \in \mathbb{Z}_{\ell}[x], \quad \operatorname{deg} h=n-2, \quad \operatorname{deg} v=2
$$

such that

$$
u(x)=v(x) h(x)
$$

and

$$
\bar{h}(x)=h(x) \bmod \ell, \quad(x-\gamma)^{2}=v(x) \bmod \ell .
$$

This implies that the splitting field $\mathbb{Q}_{\ell}\left(\Re_{h}\right)$ of $h(x)$ (over $\left.\mathbb{Q}_{\ell}\right)$ is an unramified extension of $\mathbb{Q}_{\ell}$ while the splitting field $\mathbb{Q}_{\ell}\left(\Re_{u}\right)$ of $u(x)$ (over $\left.\mathbb{Q}_{\ell}\right)$ is obtained from $\mathbb{Q}_{\ell}\left(\Re_{h}\right)$ by adjoining to it two (distinct) roots say, $\alpha_{1}$ and $\alpha_{2}$ of quadratic $v(x)$. Clearly, $\mathbb{Q}_{\ell}\left(\Re_{u}\right)$ either coincides with $\mathbb{Q}_{\ell}\left(\Re_{h}\right)$ or with a certain quadratic extension of $\mathbb{Q}_{\ell}\left(\Re_{h}\right)$, ramified or unramified. It follows that the inertia subgroup $I$ of

$$
\operatorname{Gal}\left(\mathbb{Q}_{\ell}\left(\Re_{u}\right) / \mathbb{Q}_{\ell}\right) \subset \operatorname{Perm}\left(\Re_{u}\right)
$$

is either trivial or is generated by the transposition that permutes $\alpha_{1}$ and $\alpha_{2}$ (and leaves invariant every root of $h(x))$. In the former case $\mathbb{Q}\left(\Re_{u}\right) / \mathbb{Q}$ is unramified at $\ell$ while in the latter one an inertia subgroup in

$$
\operatorname{Gal}\left(\mathbb{Q}\left(\Re_{u}\right) / \mathbb{Q}\right) \subset \operatorname{Perm}\left(\Re_{u}\right)
$$

that corresponds to $\ell$ is generated by a transposition. However, the permutation subgroup $\operatorname{Gal}\left(\mathbb{Q}\left(\Re_{u}\right) / \mathbb{Q}(\sqrt{\Delta(u)})\right)$ does not contain transpositions (see 1.1). This implies that $\mathbb{Q}\left(\Re_{u}\right) / \mathbb{Q}(\sqrt{\Delta(u)})$ is unramified at all prime divisors of $\ell$.

Example 3.4 Let us consider the polynomial

$$
u(x)=u_{n,-1,-1}(x)=x^{n}-x-1 \in \mathbb{Q}[x]
$$

over the field $K=\mathbb{Q}$. Here $B=C=-1$ and the conditions of Lemma 3.1 hold for all primes $\ell$. It is known that $u(x)$ is irreducible [13], its Galois group over $\mathbb{Q}$ is $\mathbf{S}_{n}[11$, Corollary 3, p. 233] and there exists a prime $\ell$ such that $u(x) \bmod \ell$ acquires a multiple root [15, Remark 2, p. 42]. Clearly, the discriminant $\Delta(u)=\operatorname{Discr}(n,-1,-1)$ of

\footnotetext{
${ }^{1}$ Compare with [11, Lemma 1, p. 231].
} 
$u(x)$ is an odd integer and therefore such an $\ell$ is $o d d$. It follows from Lemma 3.1 that $u(x) \bmod \ell$ has exactly one multiple root and its multiplicity is 2 .

Let $n=2 g+1$ be an odd integer greater than or equal to 5 and

$$
u(x)=u_{2 g+1,-1,-1}(x)=x^{2 g+1}-x-1 \in \mathbb{Q}[x] .
$$

Let us consider the $g$-dimensional jacobian $J\left(C_{u}\right)$ of the hyperelliptic curve $C_{u}: y^{2}=$ $x^{2 g+1}-x-1$. Since $\operatorname{Gal}(u / \mathbb{Q})=\mathbf{S}_{2 g+1}$, Theorem 1.2 tells us that $\operatorname{End}\left(J\left(C_{u}\right)\right)=\mathbb{Z}$. Now the same arguments as in Sect. 2 prove that

(i) For all primes $\ell$ the image

$$
\rho_{\ell, u}(\operatorname{Gal}(\mathbb{Q})) \subset \mathrm{Gp}\left(T_{\ell}\left(J\left(C_{u}\right)\right), e_{\ell}\right)
$$

is an open subgroup of finite index in $\operatorname{Gp}\left(T_{\ell}\left(J\left(C_{u}\right)\right), e_{\ell}\right)$.

(ii) Let $L$ be a number field and $\operatorname{Gal}(L)$ be its absolute Galois group, which we view as an open subgroup of finite index in $\operatorname{Gal}(\mathbb{Q})$. Then for all but finitely many primes $\ell$ the image

$$
\rho_{\ell, u}(\operatorname{Gal}(L)) \subset \operatorname{Gp}\left(T_{\ell}\left(J\left(C_{u}\right)\right), e_{\ell}\right)
$$

coincides with $\operatorname{Gp}\left(T_{\ell}\left(J\left(C_{u}\right)\right), e_{\ell}\right)$.

Corollary 3.5 (Corollary to Lemma 3.1) Let

$$
u(x)=u_{n, B, C}(x)=x^{n}+B x+C \in \mathbb{Z}[x]
$$

be a monic polynomial of degree $n>1$ without multiple roots such that both $B$ and $C$ are nonzero integers that enjoy the following properties:

- $(B, C)$ is either 1 or a power of 2 ,

- $(n, B)$ is either 1 or a power of 2 ,

- $(n-1, C)$ is either 1 or a power of 2 .

Suppose that the discriminant $D=\operatorname{Discr}(n, B, C)=2^{2 M} \cdot D_{0}$ where $M$ is a nonnegative integer and $D_{0}$ is an integer such that $D_{0} \equiv 1 \bmod 4$. Assume also that $D$ is not a square. Then:

(a) The quadratic extension $\mathbb{Q}(\sqrt{D}) / \mathbb{Q}$ is unramified at 2. For all odd primes $\ell$ the Galois extension $\mathbb{Q}\left(\Re_{u}\right) / \mathbb{Q}(\sqrt{D})$ is unramified at every prime divisor of $\ell$.

(b) There exists an odd prime $\ell$ that enjoys the following properties:

(i) $\ell$ divides $D_{0}$ and $u(x) \bmod \ell \in \mathbb{F}_{\ell}[x]$ has exactly one multiple root and its multiplicity is 2 . In addition, this root lies in $\mathbb{F}_{\ell}$.

(ii) The field extension $\mathbb{Q}\left(\Re_{u}\right) / \mathbb{Q}$ is ramified at $\ell$ and the Galois group

$$
\operatorname{Gal}\left(\mathbb{Q}\left(\Re_{u}\right) / \mathbb{Q}\right)=\operatorname{Gal}(u / \mathbb{Q}) \subset \operatorname{Perm}\left(\Re_{u}\right)
$$


contains a transposition. In particular, if $\mathrm{Gal}(u / \mathbb{Q})$ is doubly transitive then

$$
\operatorname{Gal}(u / \mathbb{Q})=\operatorname{Perm}\left(\Re_{f}\right) \cong \mathbf{S}_{n}
$$

and

$$
\operatorname{Gal}\left(\mathbb{Q}\left(\Re_{u}\right) / \mathbb{Q}(\sqrt{D})\right)=\mathbf{A}_{n}
$$

Proof Clearly, $D_{0}$ is not a square and

$$
\mathbb{Q}(\sqrt{D})=\mathbb{Q}\left(\sqrt{D_{0}}\right)
$$

is a quadratic field. Since $D_{0}$ is congruent to 1 modulo 4 , the quadratic extension $\mathbb{Q}\left(\sqrt{D_{0}}\right) / \mathbb{Q}$ is unramified at 2 , which proves the first assertion of (a). The conditions of Lemma 3.1 (II) hold for all odd primes $\ell$. Now the second assertion of (a) follows from Remark 3.3 and Lemma 3.1 (II)(c).

Let us start to prove (b). There are nonzero integers $S$ and $S_{0}$ such that $D_{0}=S^{2} S_{0}$ and $S_{0}$ is square-free. Clearly, both $S$ and $S_{0}$ are odd. Since

$$
D=2^{2 M} \cdot D_{0}=2^{2 M} \cdot S^{2} S_{0}=\left(2^{M} S\right)^{2} S_{0}
$$

is not a square, $S_{0} \neq 1$. Since $S$ is odd, $S^{2} \equiv 1 \bmod 4$. Since $D_{0} \equiv 1 \bmod 4$, we obtain that $S_{0} \equiv 1 \bmod 4$. It follows that $S_{0} \neq-1$. We already know that $S_{0} \neq 1$. This implies that there is a prime $\ell$ that divides $S_{0}$. Since $S_{0}$ is odd and square-free, $\ell$ is also odd and $\ell^{2}$ does not divide $S_{0}$. Let $T$ be the nonnegative integer such that $\ell^{T} \| S$. Then $\ell^{2 T+1} \| 2^{2 M} S^{2} S_{0}$, and therefore $\ell^{2 T+1} \| D$. This implies that the quadratic field extension $\mathbb{Q}(\sqrt{D}) / \mathbb{Q}$ is ramified at $\ell$. Since

$$
\mathbb{Q} \subset \mathbb{Q}(\sqrt{D}) \subset \mathbb{Q}\left(\Re_{u}\right),
$$

the field extension $\mathbb{Q}\left(\Re_{u}\right) / \mathbb{Q}$ is also ramified at $\ell$. Since $\ell \mid D$, the polynomial $u(x) \bmod \ell \in \mathbb{F}_{\ell}[x]$ has a multiple root. Now the result follows from Lemma 3.1 combined with Remark 1.10.

\section{Discriminants of Mori trinomials}

Let

$$
f(x)=f_{g, p, b, c}(x)=x^{2 g+1}-b x-\frac{p c}{4}
$$

be a Mori trinomial. Following Mori [8], let us consider the polynomial

$$
\mathbf{u}(x)=2^{2 g+1} f\left(\frac{x}{2}\right)=x^{2 g+1}-2^{2 g} b x-2^{2 g-1} p c=u_{n, B, C}(x) \in \mathbb{Z}[x] \subset \mathbb{Q}[x]
$$

with $n=2 g+1, B=-2^{2 g} b, C=-2^{2 g-1} p c$. 
Remark 4.1 Clearly, $f(x)$ and $\mathbf{u}(x)$ have the same splitting field and Galois group.

It is also clear that

$$
\Delta(\mathbf{u})=2^{(2 g+1) 2 g} \cdot \Delta(f)=\left[2^{(2 g+1) g}\right]^{2} \cdot \Delta(f) .
$$

In particular, $\Delta(\mathbf{u})$ is not a square, thanks to Remark 1.6.

- By Theorem 1.4(i, iii), the polynomial $f(x)$ is irreducible over $\mathbb{Q}$ and its Galois group is doubly transitive. This implies that $\mathbf{u}(x)$ is irreducible over $\mathbb{Q}$ and its Galois group over $\mathbb{Q}$ is also doubly transitive. (See also Theorem 6.6 (i, ii) below.)

- For all $g$ the hyperelliptic curves $C_{f}$ and $C_{\mathbf{u}}$ are biregularly isomorphic over $\mathbb{Q}(\sqrt{2})$. It follows that the jacobians $J\left(C_{\mathbf{u}}\right)$ and $J\left(C_{f}\right)$ are also isomorphic over $\mathbb{Q}(\sqrt{2})$. In particular, End $\left(J\left(C_{\mathbf{u}}\right)\right)=\operatorname{End}\left(J\left(C_{f}\right)\right)$.

Clearly, the conditions of Lemma 3.1 hold for $u(x)=\mathbf{u}(x)$ for all odd primes $\ell$. The discriminant $\Delta(\mathbf{u})$ of $\mathbf{u}(x)$ coincides with

$$
\begin{aligned}
\operatorname{Discr}(n, B, C)=(-1)^{(2 g+1) 2 g / 2} & (2 g+1)^{2 g+1}\left[-2^{2 g-1} p c\right]^{2 g} \\
& +(-1)^{2 g(2 g-1) / 2}(2 g)^{2 g}\left[-2^{2 g} b\right]^{2 g+1} .
\end{aligned}
$$

It follows that

$$
\Delta(\mathbf{u})=(-1)^{g} 2^{2 g(2 g-1)}\left[(2 g+1)^{2 g+1}(p c)^{2 g}-2^{6 g} g^{2 g} b^{2 g+1}\right] .
$$

This implies that

$$
\Delta(\mathbf{u})=2^{2[g(2 g-1)]} D_{0},
$$

where

$$
D_{0}=(-1)^{g}\left\{(2 g+1)\left[(2 g+1)^{g}(p c)^{g}\right]^{2}-2^{6 g} g^{2 g} b^{2 g+1}\right\} \text {. }
$$

Clearly, $D_{0}$ is an odd integer that is not divisible by $p$. It is also clear that $D_{0}$ is congruent to $(-1)^{g}(2 g+1)$ modulo 4 (because every odd square is congruent to 1 modulo 4). This implies that

$$
D_{0} \equiv 1 \bmod 4
$$

for all $g$.

\section{Proof of Theorem 1.7}

Let us apply Lemma 3.1 (II) to

$$
\mathbf{u}(x)=2^{2 g+1} f\left(\frac{x}{2}\right)=x^{2 g+1}-2^{2 g} b x-2^{2 g-1} p c .
$$

We obtain that for each odd prime $\ell$ the polynomial $\mathbf{u}(x) \bmod \ell \in \mathbb{F}_{\ell}[x]$ has, at most, one multiple root; in addition, this root is double and lies in $\mathbb{F}_{\ell}$. Applying to $\mathbf{u}(x)$ 
Corollary 3.5 combined with formulas (3) and (4) of Sect. 4, we conclude that there exists an odd $\ell \neq p$ such that $\mathbf{u}(x) \bmod \ell$ has exactly one multiple root; this root is double and lies in $\mathbb{F}_{\ell}$. In addition, $\operatorname{Gal}(\mathbf{u} / \mathbb{Q})$ coincides with $\mathbf{S}_{2 g+1}$, because it is doubly transitive. Now the assertions (i) and (ii) follow readily from the equality

$$
f(x) \bmod \ell=\frac{\mathbf{u}(2 x)}{2^{2 g+1}} \bmod \ell
$$

that holds for all odd primes $\ell$.

By Remark 4.1, $\operatorname{Gal}(f / \mathbb{Q})=\operatorname{Gal}(\mathbf{u} / \mathbb{Q})$ and therefore also coincides with $\mathbf{S}_{2 g+1}$, which implies (in light of 1.1) that $\operatorname{Gal}\left(\mathbb{Q}\left(\mathfrak{R}_{f}\right) / \mathbb{Q}(\sqrt{\Delta(f)})\right)=\mathbf{A}_{2 g+1}$. This proves (iii). Now Remark 1.9 implies that $\operatorname{End}\left(J\left(C_{f}\right)\right)=\mathbb{Z}$. This proves (iv). In order to prove (iii'), first notice that the Galois extension $\mathbb{Q}\left(\Re_{f}\right) / \mathbb{Q}$ is ramified at 2, Remark $1.5(\mathrm{I})$, while $\mathbb{Q}(\sqrt{\Delta(f)})=\mathbb{Q}(\sqrt{\Delta(u)})$ is unramified at 2 over $\mathbb{Q}$ in light of formulas (3) and (4) in Sect. 4 (and Corollary 3.5 (a)). This implies that $\mathbb{Q}\left(\Re_{f}\right) / \mathbb{Q}(\sqrt{\Delta(f)})$ is ramified at some prime divisor of 2 . Since all the field extensions involved are Galois, $\mathbb{Q}\left(\Re_{f}\right) / \mathbb{Q}(\sqrt{\Delta(f)})$ is actually ramified at all prime divisors of 2 . This proves the first assertion of (iii'). The second assertion of (iii') follows from Corollary 3.5 (a). This proves (iii').

\section{Variants and complements}

Throughout this section, $K$ is a number field. We write $\mathcal{O}$ for the ring of integers in $K$. If $\mathfrak{b}$ is a maximal ideal in $\mathcal{O}$ then we write $k(\mathfrak{b})$ for the (finite) residue field $\mathcal{O} / \mathfrak{b}$. As usual, we call char $(k(\mathfrak{b}))$ the residual characteristic of $\mathfrak{b}$. We write $K_{\mathfrak{b}}$ for the $\mathfrak{b}$-adic completion of $K$ and

$$
\mathcal{O}_{\mathfrak{b}} \subset K_{\mathfrak{b}}
$$

for the ring of $\mathfrak{b}$-adic integers in the field $K_{\mathfrak{b}}$. We consider the subring $\mathcal{O}[1 / 2] \subset K$ generated by $1 / 2$ over $\mathcal{O}$. We have

$$
\mathcal{O} \subset \mathcal{O}\left[\frac{1}{2}\right] \subset K
$$

If $\mathfrak{b} \subset \mathcal{O}$ is a maximal ideal in $\mathcal{O}$ with odd residual characteristic then

$$
\mathcal{O} \subset \mathcal{O}\left[\frac{1}{2}\right] \subset \mathcal{O}_{\mathfrak{b}}
$$

the ideal $\mathfrak{b} \mathcal{O}[1 / 2]$ is a maximal ideal in $\mathcal{O}[1 / 2]$ and

$$
k(\mathfrak{b})=\mathcal{O} / \mathfrak{b}=\mathcal{O}\left[\frac{1}{2}\right] / \mathfrak{b} \mathcal{O}\left[\frac{1}{2}\right]=\mathcal{O}_{\mathfrak{b}} / \mathfrak{b} \mathcal{O}_{\mathfrak{b}}
$$

Lemma 3.1 (II) and its proof admit the following straightforward generalization. 


\section{Lemma 6.1 Let}

$$
u(x)=u_{n, B, C}(x)=x^{n}+B x+C \in \mathcal{O}[x]
$$

be a monic polynomial of degree $n>1$ such that $B \neq 0$ and $C \neq 0$. Let $\mathfrak{b}$ be a maximal ideal in $\mathcal{O}$ that enjoys the following properties:

(i) $B \mathcal{O}+C \mathcal{O}+\mathfrak{b}=\mathcal{O}$,

(ii) $n \mathcal{O}+B \mathcal{O}+\mathfrak{b}=\mathcal{O}$,

(iii) $(n-1) \mathcal{O}+C \mathcal{O}+\mathfrak{b}=\mathcal{O}$.

Suppose that $u(x)$ has no multiple roots. Let us consider the polynomial

$$
\bar{u}(x)=u(x) \bmod \mathfrak{b} \in k(\mathfrak{b})[x] .
$$

Then:

(a) $\bar{u}(x)$ has, at most, one multiple root in an algebraic closure of $k(\mathfrak{b})$.

(b) If such a multiple root say, $\gamma$, does exist, then $n(n-1) B C \notin \mathfrak{b}$ and $\gamma$ is a double root of $\bar{u}(x)$. In addition, $\gamma$ is a nonzero element of $k(\mathfrak{b})$.

(c) If such a multiple root does exist then either the field extension $K\left(\Re_{u}\right) / K$ is unramified at $\mathfrak{b}$ or a corresponding inertia subgroup at $\mathfrak{b}$ in

$$
\operatorname{Gal}\left(K\left(\Re_{u}\right) / K\right)=\operatorname{Gal}(u / \mathrm{K}) \subset \operatorname{Perm}\left(\Re_{u}\right)
$$

is generated by a transposition. In both cases the Galois extension $K\left(\Re_{u}\right) /$ $K(\sqrt{\Delta(u)})$ is unramified at all prime divisors of $\mathfrak{b}$.

Remark 6.2 In the notation of Lemma 6.1, suppose that $\bar{u}(x)$ has no multiple roots, i.e., $\Delta(u) \notin \mathfrak{b}$. Then clearly the Galois extension $K\left(\Re_{u}\right) / K$ is unramified at $\mathfrak{b}$.

Proof We have

$$
\bar{u}(x)=x^{n}+\bar{B} x+\bar{C} \in k(\mathfrak{b})[x],
$$

where

$$
\bar{B}=B \bmod \mathfrak{b} \in k(\mathfrak{b}), \quad \bar{C}=C \bmod \mathfrak{b} \in k(\mathfrak{b}) .
$$

The condition (i) implies that either $\bar{B} \neq 0$ or $\bar{C} \neq 0$. The condition (ii) implies that if $\bar{B}=0$ then $n \neq 0$ in $k(\mathfrak{b})$. It follows that if $\bar{B}=0$ then $n \bar{C} \neq 0$.

The condition (iii) implies that if $n-1=0$ in $k(\mathfrak{b})$ then $\bar{C} \neq 0$ (and, of course, $n \neq 0$ in $k(\mathfrak{b}))$. On the other hand, if $\bar{C}=0$ then $n-1 \neq 0$ in $k(\mathfrak{b})$.

Suppose $\bar{u}(x)$ has a multiple root $\gamma$ in an algebraic closure of $k(\mathfrak{b})$. Then as in the proof of Lemma 3.1 (II),

$$
\Delta(\bar{u})=(-1)^{n(n-1) / 2} n^{n} \bar{C}^{n-1}+(-1)^{(n-1)(n-2) / 2}(n-1)^{n-1} \bar{B}^{n}=0 .
$$


This implies that

$$
n^{n} \bar{C}^{n-1}= \pm(n-1)^{n-1} \bar{B}^{n} .
$$

This implies that if $n-1=0$ in $k(\mathfrak{b})$ then $\bar{C}=0$, which is not the case. This proves that $n-1 \neq 0$ in $k(\mathfrak{b})$. On the other hand, if $\bar{B}=0$ then $\bar{C} \neq 0$ and $n \neq 0$ in $k(\mathfrak{b})$. Then (5) implies that $\bar{C}=0$ and we get a contradiction that proves that $\bar{B} \neq 0$. If $n=0$ in $k(\mathfrak{b})$ then $n-1 \neq 0$ in $k(\mathfrak{b})$ and (5) implies that $\bar{B}=0$, which is not the case. The obtained contradiction proves that $n \neq 0$ in $k(\mathfrak{b})$. If $\bar{C}=0$ then (5) implies that $\bar{B}=0$, which is not the case. This proves that the maximal ideal $\mathfrak{b}$ does not contain $n(n-1) B C$.

On the other hand, we have as in the proof of Lemma 3.1 (II) that

$$
x \cdot \bar{u}^{\prime}(x)-n \cdot \bar{u}(x)=-(n-1) \bar{B} x-n \bar{C}
$$

and therefore $-(n-1) \bar{B} \gamma-n \bar{C}=0$. It follows that

$$
\gamma=-\frac{n \bar{C}}{(n-1) \bar{B}}
$$

is a nonzero element of $k(\mathfrak{b})$. The second derivative $\bar{u}^{\prime \prime}(x)=n(n-1) x^{n-2}$ and

$$
\bar{u}^{\prime \prime}(\gamma)=n(n-1) \gamma^{n-2} \neq 0 .
$$

It follows that $\gamma$ is a double root of $\bar{u}(x)$. This proves (a) and (b).

In order to prove (c), notice that as in the proof of Lemma 3.1 (II)(c), there exists a monic degree $n-2$ polynomial $\bar{h}(x) \in k(\mathfrak{b})[x]$ such that

$$
\bar{u}(x)=(x-\gamma)^{2} \cdot \bar{h}(x)
$$

and $\bar{h}(x)$ and $(x-\gamma)^{2}$ are relatively prime. By Hensel's Lemma, there exist monic polynomials

$$
h(x), v(x) \in \mathcal{O}_{\mathfrak{b}}[x], \quad \operatorname{deg} h=n-2, \quad \operatorname{deg} v=2
$$

such that

$$
u(x)=v(x) h(x)
$$

and

$$
\bar{h}(x)=h(x) \bmod \mathfrak{b}, \quad(x-\gamma)^{2}=v(x) \bmod \mathfrak{b} .
$$

This implies that the splitting field $K_{\mathfrak{b}}\left(\Re_{h}\right)$ of $h(x)$ (over $\left.K_{\mathfrak{b}}\right)$ is an unramified extension of $\mathrm{K}_{\mathfrak{b}}$ while the splitting field $\mathrm{K}_{\mathfrak{b}}\left(\Re_{u}\right)$ of $u(x)$ (over $\left.\mathrm{K}_{\mathfrak{b}}\right)$ is obtained from $\mathrm{K}_{\mathfrak{b}}\left(\Re_{h}\right)$ by adjoining to it two (distinct) roots say, $\alpha_{1}$ and $\alpha_{2}$ of quadratic $v(x)$. The field $K_{\mathfrak{b}}\left(\Re_{u}\right)$ 
coincides either with $K_{\mathfrak{b}}\left(\Re_{h}\right)$ or with a certain quadratic extension of $K_{\mathfrak{b}}\left(\Re_{h}\right)$, ramified or unramified. It follows that the inertia subgroup $I$ of

$$
\operatorname{Gal}\left(K_{\mathfrak{b}}\left(\Re_{u}\right) / K_{\mathfrak{b}}\right) \subset \operatorname{Perm}\left(\Re_{u}\right)
$$

is either trivial or is generated by the transposition that permutes $\alpha_{1}$ and $\alpha_{2}$ (and leaves invariant every root of $h(x))$. In the former case $K\left(\Re_{u}\right) / K$ is unramified at $\mathfrak{b}$ while in the latter one an inertia subgroup in

$$
\operatorname{Gal}\left(K\left(\Re_{u}\right) / K\right) \subset \operatorname{Perm}\left(\Re_{u}\right)
$$

that corresponds to $\mathfrak{b}$ is generated by a transposition. In both cases the Galois (sub)group $\operatorname{Gal}\left(K\left(\Re_{u}\right) / K(\sqrt{\Delta(u)})\right)$ does not contain transpositions (see 1.1). This implies that $K\left(\mathfrak{R}_{u}\right) / K(\sqrt{\Delta(u)})$ is unramified at all prime divisors of $\mathfrak{b}$.

Corollary 3.5 admits the following partial generalization.

Lemma 6.3 Let $K$ be a number field and $\mathcal{O}$ be its ring of integers. Let

$$
u(x)=u_{n, B, C}(x)=x^{n}+B x+C \in \mathcal{O}[x]
$$

be a monic polynomial without multiple roots of degree $n>1$ such that both $B$ and $C$ are not zeros. Suppose that there is a nonnegative integer $N$ such that

$$
2^{N} \mathcal{O} \subset B \mathcal{O}+C \mathcal{O}, \quad 2^{N} \mathcal{O} \subset n \mathcal{O}+B \mathcal{O}, \quad 2^{N} \mathcal{O} \subset(n-1) \mathcal{O}+C \mathcal{O} .
$$

Suppose that there is a nonnegative integer $M$ such that the discriminant $D=\Delta(u)=$ $2^{2 M} \cdot D_{0}$ with $D_{0} \in \mathcal{O}$. Assume also that $D, D_{0}$ and $K$ enjoy the following properties:

(i) $D$ is not a square in $K$ and $D_{0}-1 \in 4 \mathcal{O}$.

(ii) The class number of $K$ is odd (e.g., $\mathcal{O}$ is a principal ideal domain).

(iii) Either $K$ is totally imaginary, i.e., it does not admit an embedding into the field of real numbers or $K$ is totally real and $D_{0}$ is totally positive.

Then:

(a) The quadratic extension $K(\sqrt{\Delta(u)}) / K$ is unramified at every prime divisor of 2 . The Galois extension $K\left(\Re_{u}\right) / K(\sqrt{\Delta(u)})$ is unramified at every prime ideal $\mathfrak{b}$ of odd residual characteristic.

(b) There exists a maximal ideal $\mathfrak{b} \subset \mathcal{O}$ with residue field $k(\mathfrak{b})$ of odd characteristic that enjoys the following properties:

- $D_{0} \in \mathfrak{b}$, the polynomial $u(x) \mathfrak{b} \bmod \in k(\mathfrak{b})[x]$ has exactly one multiple root and its multiplicity is 2 . In addition, this root lies in $k(\mathfrak{b})$.

- The field extension $K\left(\mathfrak{R}_{u}\right) / K$ is ramified at $\mathfrak{b}$ and the Galois group

$$
\operatorname{Gal}\left(K\left(\Re_{u}\right) / K\right)=\operatorname{Gal}(u / \mathrm{K}) \subset \operatorname{Perm}\left(\Re_{u}\right)
$$


contains a transposition. In particular, if $\mathrm{Gal}(u / \mathrm{K})$ is doubly transitive then

$$
\operatorname{Gal}(u / \mathrm{K})=\operatorname{Perm}\left(\Re_{f}\right) \cong \mathbf{S}_{n}
$$

and

$$
\operatorname{Gal}\left(K\left(\Re_{u}\right) / K(\sqrt{\Delta(u)})\right)=\mathbf{A}_{n} .
$$

Proof Let us prove (a). Clearly,

$$
E=K\left(\sqrt{D_{0}}\right)=K(\sqrt{D})=K(\sqrt{\Delta(u)}) \subset K\left(\Re_{u}\right)
$$

is a quadratic extension of $K$. Notice that $\theta=\left(1+\sqrt{D_{0}}\right) / 2 \in E$ is a root of the quadratic equation

$$
v_{2}(x)=x^{2}-x+\frac{1-D_{0}}{4} \in \mathcal{O}[x]
$$

and therefore is an algebraic integer. In addition, $E=K(\theta)$.

If a maximal ideal $\mathfrak{b}_{2}$ in $\mathcal{O}$ has residual characteristic 2 then the quadratic polynomial

$$
v_{2}(x) \bmod \mathfrak{b}_{2}=x^{2}-x+\left(\frac{1-D_{0}}{4}\right) \bmod \mathfrak{b}_{2} \in k\left(\mathfrak{b}_{2}\right)[x]
$$

has no multiple roots, because its derivative is a nonzero constant -1 . This implies that $E / K$ is unramified at all prime divisors of 2. On the other hand, the conditions of Lemma 6.1 hold for all maximal ideals $\mathfrak{b}$ of $\mathcal{O}$ with odd residual characteristic. Now Remark 6.2 and Lemma 6.1 (c) imply that the Galois extension $K\left(\Re_{u}\right) / K(\sqrt{\Delta(u)})$ is unramified at every $\mathfrak{b}$ of odd residual characteristic. This proves (a).

In order to prove (b), notice that the condition (iii) implies that either all archimedean places of both $E$ and $K$ are complex or all archimedean places of both $E$ and $K$ are real. This implies that $E / K$ is unramified at all infinite primes. Since the class number of $K$ is odd, the classical results about Hilbert class fields [6, Chapter 2, Section 1.2] imply that there is a maximal ideal $\mathfrak{b} \subset \mathcal{O}$ such that $E / K=K(\sqrt{D}) / K$ is ramified at $\mathfrak{b}$. Since $E / K$ is unramified at all prime divisors of 2, the residual characteristic of $\mathfrak{b}$ is $o d d$, i.e., $2 \notin \mathfrak{b}$. This implies that

$$
\Delta(u)=D \in \mathfrak{b} .
$$

Since $D=2^{2 M} \cdot D_{0}$ and $\mathfrak{b}$ is a prime (actually, maximal) ideal in $\mathcal{O}$, we have $D_{0} \in \mathfrak{b}$. It also follows that

$$
u(x) \bmod \mathfrak{b} \in k(\mathfrak{b})[x]
$$

has a multiple root. Now we are in a position to apply Lemma 6.1. Since $K\left(\Re_{u}\right) \supset E$, the field extension $K\left(\Re_{u}\right) / K$ is ramified at $\mathfrak{b}$. Applying Lemma 6.1, we conclude 
that $u(x) \bmod \mathfrak{b}$ has exactly one multiple root, this root is double and lies in $k(\mathfrak{b})$. In addition,

$$
\operatorname{Gal}\left(K\left(\Re_{u}\right) / K\right) \subset \operatorname{Perm}\left(\Re_{u}\right)
$$

contains a transposition. This implies that if $\operatorname{Gal}\left(K\left(\Re_{u}\right) / K\right)$ is doubly transitive then $\operatorname{Gal}\left(K\left(\Re_{u}\right) / K\right)$ coincides with Perm $\left(\Re_{u}\right) \cong \mathbf{S}_{n}$. Of course, this implies that $\operatorname{Gal}\left(K\left(\Re_{u}\right) / K(\sqrt{\Delta(u)})\right)=\mathbf{A}_{n}$.

6.4 Generalized Mori quadruples Let us consider a quadruple $(g, \mathfrak{p}, \mathbf{b}, \mathbf{c})$ where $g$ is a positive integer, $\mathfrak{p}$ is a maximal ideal in $\mathcal{O}$ while $\mathbf{b}$ and $\mathbf{c}$ are elements of $\mathcal{O}$ that enjoy the following properties:

- The residue field $k(\mathfrak{p})=\mathcal{O} / \mathfrak{p}$ is a finite field of odd characteristic. If $q$ is the cardinality of $k(\mathfrak{p})$ then every prime divisor of $g$ is also a divisor of $(q-1) / 2$. In particular, if $g$ is even then $q-1$ is divisible by 4 .

- The residue $\mathbf{b} \bmod \mathfrak{p}$ is a primitive element of $k(\mathfrak{p})$, i.e., it has multiplicative order $q-1$. In particular,

$$
\mathbf{b} \mathcal{O}+\mathfrak{p}=\mathcal{O} .
$$

The conditions (i) and (ii) imply that for each prime divisor $d$ of $g$ the residue $\mathbf{b} \bmod \mathfrak{p}$ is not a $d$ th power in $k(\mathfrak{p})$. Since $q-1$ is even, $\mathbf{b} \bmod \mathfrak{p}$ is not a square in $k(\mathfrak{p})$. So, if $d$ is a prime divisor of $2 g$ then $\mathbf{b} \bmod \mathfrak{p}$ is not a $d$ th power in $k(\mathfrak{p})$. If $2 g$ is divisible by 4 then $g$ is even and $q-1$ is divisible by 4 , i.e., -1 is a square in $k(\mathfrak{p})$. It follows that $-4 \mathbf{b} \bmod \mathfrak{p}$ is not a square in $k(\mathfrak{p})$. Thanks to [7, Thereom 9.1, Chapter VI, Section 9], the last two assertions imply that the polynomial

$$
x^{2 g}-\mathbf{b} \bmod \mathfrak{p} \in k(\mathfrak{p})[x]
$$

is irreducible over $k(\mathfrak{p})$. This implies that its Galois group over (the finite field) $k(\mathfrak{p})$ is an order $2 g$ cyclic group.

- $\mathbf{c} \in \mathfrak{p}, \mathbf{c}-1 \in 2 \mathcal{O}$ and

$$
\mathcal{O}=\mathbf{b} \mathcal{O}+\mathbf{c} \mathcal{O}=\mathbf{b} \mathcal{O}+(2 g+1) \mathcal{O}=2 g \mathcal{O}+\mathbf{c} \mathcal{O} .
$$

We call such a quadruple a generalized Mori quadruple (in $K$ ).

Example 6.5 Suppose that $K$ and $g$ are given. By Dirichlet's Theorem about primes in arithmetic progressions, there is a prime $p$ that does not divide $2 g+1$ and is congruent to 1 modulo $2 g$. (In fact, there are infinitely many such primes.) Clearly, $p$ is odd. Let us choose a maximal ideal $\mathfrak{p}$ of $\mathcal{O}$ that contains $p$ and denote by $q$ the cardinality of the finite residue field $k(\mathfrak{p})$. Then $\operatorname{char}(k(\mathfrak{p}))=p$ and $q$ is a power of $p$. This implies that $q-1$ is divisible by $p-1$ and therefore is divisible by $2 g$. Let us choose a generator $\widetilde{\mathbf{b}} \in k(\mathfrak{p})$ of the multiplicative cyclic group $k(\mathfrak{p})^{*}$. Let $r$ be a nonzero integer that is 
relatively prime to $2 g+1$. (E.g., $r= \pm 1, \pm 2$.) Using Chinese Remainder Theorem, one may find $\mathbf{b} \in \mathcal{O}$ such that

$$
\mathbf{b} \bmod \mathfrak{p}=\widetilde{\mathbf{b}}, \quad \mathbf{b}-r \in(2 g+1) \mathcal{O} .
$$

(Clearly, $\mathbf{b} \notin \mathfrak{p}$.) Now the same theorem allows us to find $\mathbf{c} \in \mathfrak{p} \subset \mathcal{O}$ such that $\mathbf{c}-1 \in 2 g \mathbf{b} \mathcal{O}$. Then $(g, \mathfrak{p}, \mathbf{b}, \mathbf{c})$ is a generalized Mori quadruple in $K$.

Let us consider the polynomials

$$
F(x)=F_{g, \mathfrak{p}, \mathbf{b}, \mathbf{c}}(x)=x^{2 g+1}-\mathbf{b} x-\frac{\mathbf{c}}{4} \in \mathcal{O}\left[\frac{1}{2}\right][x] \subset K[x]
$$

and

$$
U(x)=2^{2 g+1} F\left(\frac{x}{2}\right)=x^{2 g+1}-2^{2 g} \mathbf{b} x-2^{2 g-1} \mathbf{c} \in \mathcal{O}[x] \subset K[x] .
$$

Theorem 6.6 Let $(g, \mathfrak{p}, \mathbf{b}, \mathbf{c})$ be a generalized Mori quadruple in K. Assume also that there exists a maximal ideal $\mathfrak{b}_{2} \subset \mathcal{O}$ of residual characteristic 2 such that the ramification index e $\left(\mathfrak{b}_{2}\right)$ of $\mathfrak{b}_{2}$ (over 2 ) in $K / \mathbb{Q}$ is relatively prime to $2 g+1$. Then:

(i) The polynomial $F(x)=F_{g, \mathfrak{p}, \mathbf{b}, \mathbf{c}}(x) \in K[x]$ is irreducible over $K_{\mathfrak{b}_{2}}$ and therefore over $K$. In addition, the Galois extension $K\left(\mathfrak{R}_{F}\right) / K$ is ramified at $\mathfrak{b}_{2}$.

(ii) The transitive Galois group

$$
\operatorname{Gal}(F / \mathrm{K})=\operatorname{Gal}\left(K\left(\Re_{F}\right) / K\right) \subset \operatorname{Perm}\left(\mathfrak{R}_{F}\right)=\mathbf{S}_{2 g+1}
$$

contains a cycle of length $2 g$. In particular, $\mathrm{Gal}(F / \mathrm{K})$ is doubly transitive and is not contained in $\mathbf{A}_{2 g+1}$, and $\Delta(F)$ is not a square in $K$.

(iii) Assume that $K$ is a totally imaginary number field with odd class number. Then $\operatorname{Gal}(F / K)=\operatorname{Perm}\left(\mathfrak{R}_{F}\right)$. If, in addition, $g>1$ then $\operatorname{End}\left(J\left(C_{F}\right)\right)=\mathbb{Z}$.

(iv) Assume that $K$ is a totally imaginary number field with odd class number and $g>1$. Then:

- For all primes $\ell$ the image $\rho_{\ell, F}(\mathrm{Gal}(K))$ is an open subgroup of finite index in $\mathrm{Gp}\left(T_{\ell}\left(J\left(C_{F}\right)\right), e_{\ell}\right)$.

- Let $L$ be a number field that contains $K$ and $\mathrm{Gal}(L)$ be the absolute Galois group of $L$, which we view as an open subgroup of finite index in $\operatorname{Gal}(L)$. Then for all but finitely many primes $\ell$ the image $\rho_{\ell, F}(\mathrm{Gal}(L))$ coincides with $\operatorname{Gp}\left(T_{\ell}\left(J\left(C_{F}\right)\right), e_{\ell}\right)$.

Remark 6.7 If $K$ is a quadratic field then for every maximal ideal $\mathfrak{b}_{2} \subset \mathcal{O}$ (with residual characteristic 2$)$ the ramification index $e\left(\mathfrak{b}_{2}\right)$ of $\mathfrak{b}_{2}$ in $K / \mathbb{Q}$ is either 1 or 2 : in both cases it is relatively prime to odd $2 g+1$. This implies that if $K$ is an imaginary quadratic field with odd class number then all conclusions of Theorem 6.6 hold for every generalized Mori quadruple $(g, \mathfrak{p}, \mathbf{b}, \mathbf{c})$. In particular, the Galois extension $K\left(\Re_{F}\right) / K$ is ramified at every $\mathfrak{b}_{2}$. 
One may find the list of imaginary quadratic fields with small, $\leq 23$, odd class number in [1, pp.322-324]; see also [16, Table 4, p.936].

Proof of Theorem 6.6 The $\mathfrak{b}_{2}$-adic Newton polygon of $F(x)$ consists of one segment that connects the points $\left(0,-2 e\left(\mathfrak{b}_{2}\right)\right)$ and $(2 g+1,0)$, which are its only integer points, because $e\left(\mathfrak{b}_{2}\right)$ and $2 g+1$ are relatively prime and therefore $2 e\left(\mathfrak{b}_{2}\right)$ and $2 g+1$ are relatively prime. Now the irreducibility of $F(x)$ over $K_{\mathfrak{b}_{2}}$ follows from EisensteinDumas Criterion [9, Corollary 3.6, p. 316], [4, p. 502]. This proves (i). It also proves that the Galois extension $K\left(\mathfrak{R}_{F}\right) / K$ is ramified at $\mathfrak{b}_{2}$.

In order to prove (ii), let us consider the reduction

$$
\widetilde{F}(x)=F(x) \bmod \mathfrak{p} \odot\left[\frac{1}{2}\right]=x^{2 g+1}-\widetilde{\mathbf{b}} x \in k(\mathfrak{p})[x]
$$

where $\widetilde{\mathbf{b}}=\mathbf{b} \bmod \mathfrak{p} \in k(\mathfrak{p})$. So,

$$
\widetilde{F}(x)=x\left(x^{2 g}-\widetilde{\mathbf{b}}\right) \in k(\mathfrak{p})[x] .
$$

We have already seen in 6.4 that $x^{2 g}-\widetilde{\mathbf{b}}$ is irreducible over $k(\mathfrak{p})$ and its Galois group is an order $2 g$ cyclic group. We also know that $\widetilde{\mathbf{b}} \neq 0$ and therefore the polynomials $x$ and $x^{2 g}-\widetilde{\mathbf{b}}$ are relatively prime. This implies that $K\left(\Re_{F}\right) / K$ is unramified at $\mathfrak{p}$ and a corresponding Frobenius element in $\operatorname{Gal}\left(K\left(\Re_{F}\right) / K\right) \subset \operatorname{Perm}\left(\mathfrak{R}_{F}\right)$ is a cycle of length $2 g$. This proves (ii). (Compare with arguments on [8, p. 107].)

The map $\alpha \mapsto 2 \alpha$ is a $\operatorname{Gal}(K)$-equivariant bijection between the sets of roots $\mathfrak{R}_{F}$ and $\mathfrak{R}_{U}$, which induces a group isomorphism between permutation groups $\operatorname{Gal}\left(\mathfrak{R}_{F}\right) \subset \operatorname{Perm}\left(\mathfrak{R}_{F}\right)$ and $\operatorname{Gal}\left(\mathfrak{R}_{U}\right) \subset \operatorname{Perm}\left(\mathfrak{R}_{U}\right)$. In particular, the double transitivity of $\operatorname{Gal}\left(\mathfrak{R}_{F}\right)$ implies the double transitivity of $\operatorname{Gal}\left(\Re_{U}\right)$. On the other hand,

$$
\Delta(U)=2^{(2 g+1) 2 g} \Delta(F)=\left[2^{(2 g+1) g}\right]^{2} \Delta(F) .
$$

This implies that $\Delta(U)$ is not a square in $K$ as well. The discriminant $\Delta(U)$ is given by the formula, Remark 3.2,

$$
\begin{aligned}
D= & \Delta(U)=(-1)^{(2 g+1) 2 g / 2}(2 g+1)^{2 g+1}\left[-2^{2 g-1} \mathbf{c}\right]^{2 g} \\
& +(-1)^{2 g(2 g-1) / 2}(2 g)^{2 g}\left[-2^{2 g} \mathfrak{b}\right]^{2 g+1} \\
= & (-1)^{g} 2^{2 g(2 g-1)}\left[(2 g+1)^{2 g+1} \mathbf{c}^{2 g}-2^{6 g} g^{2 g} \mathfrak{b}^{2 g+1}\right] \\
= & 2^{2[g(2 g-1)]}\left\{(-1)^{g}\left[(2 g+1)^{2 g+1} \mathbf{c}^{2 g}-2^{6 g} g^{2 g} \mathfrak{b}^{2 g+1}\right]\right\} .
\end{aligned}
$$

We have $D=2^{2 M} D_{0}$, where $M=g(2 g-1)$ is a positive integer and

$$
D_{0}=(-1)^{g}\left[(2 g+1)^{2 g+1} \mathbf{c}^{2 g}-2^{6 g} g^{2 g} \mathfrak{b}^{2 g+1}\right] \in \mathcal{O} \text {. }
$$

Since $\mathbf{c}-1 \in 2 \mathcal{O}$, we have $\mathbf{c}^{2}-1 \in 4 \mathcal{O}$ and

$$
D_{0} \equiv(-1)^{g}(2 g+1)^{2 g+1} \bmod 4 \mathcal{O} .
$$


Since $(2 g+1)^{2 g}=\left[(2 g+1)^{2}\right]^{g} \equiv 1 \bmod 4$, we conclude $D_{0} \equiv(-1)^{g}(2 g+1)$ $\bmod 40$. This implies that

$$
D_{0}-1 \in 4 \mathcal{O} \text {. }
$$

Applying Lemma 6.3 to

$$
\begin{array}{r}
n=2 g+1, \quad B=-2^{2 g} \mathbf{b}, \quad C=-2^{2 g-1} \mathbf{c}, \\
u(x)=U(x), \quad M=g(2 g-1), \quad N=2 g,
\end{array}
$$

we conclude that doubly transitive $\operatorname{Gal}(U / K)$ coincides with Perm $\left(\Re_{U}\right)$ and therefore $\operatorname{Gal}(F / K)$ coincides with Perm $\left(\Re_{F}\right) \cong \mathbf{S}_{2 g+1}$. If $g>1$ then Theorem 1.2 tells us that $\operatorname{End}\left(J\left(C_{F}\right)\right)=\mathbb{Z}$. This proves (iii). We also obtain that there exists a maximal ideal $\mathfrak{b} \subset \mathcal{O}$ with odd residual characteristic such that $U(x) \bmod \mathfrak{b} \in k(\mathfrak{b})[x]$ has exactly one multiple root, this root is double and lies in $k(\mathfrak{b})$. Since

$$
F(x)=\frac{U(2 x)}{2^{2 g+1}}
$$

we obtain that

$$
F(x) \bmod \mathfrak{b} \mathcal{O}\left[\frac{1}{2}\right]=\frac{U(2 x)}{2^{2 g+1}} \bmod \mathfrak{b} \in k(\mathfrak{b})[x] .
$$

This implies that the polynomial $F(x) \bmod \mathfrak{b} \mathcal{O}[1 / 2] \in k(\mathfrak{b})[x]$ has exactly one multiple root, this root is double and lies in $k(\mathfrak{b})$. The properties of $F(x) \bmod \mathfrak{b} \mathcal{O}[1 / 2]$ imply that $J\left(C_{F}\right)$ has a semistable reduction at $\mathfrak{b}$ with toric dimension 1 . Now it follows from [21, Theorem 4.3] that for for all primes $\ell$ the image $\rho_{\ell, F}(\operatorname{Gal}(K))$ is an open subgroup of finite index in $\operatorname{Gp}\left(T_{\ell}\left(J\left(C_{F}\right)\right), e_{\ell}\right)$. It follows from [5, Theorem 1] that if $L$ is a number field containing $K$ then for all but finitely many primes $\ell$ the image $\rho_{\ell, F}(\mathrm{Gal}(L))$ coincides with $\mathrm{Gp}\left(T_{\ell}\left(J\left(C_{F}\right)\right), e_{\ell}\right)$. This proves (iv).

Corollary 6.8 We keep the notation of Theorem 6.6. Let $K$ be an imaginary quadratic field with odd class number. Let $(g, \mathfrak{p}, \mathbf{b}, \mathbf{c})$ be a generalized Mori quadruple in $K$ and $F(x)=F_{g, \mathfrak{p}, \mathbf{b}, \mathbf{c}}(x) \in K[x]$. Then

$$
\operatorname{Gal}\left(K\left(\Re_{F}\right) / K(\sqrt{\Delta(F)})\right)=\mathbf{A}_{2 g+1}
$$

and the Galois extension $K\left(\Re_{F}\right) / K(\sqrt{\Delta(F)})$ is unramified everywhere outside 2 and ramified at all prime divisors of 2 .

Proof As above, let us consider the polynomial

$$
U(x)=2^{2 g+1} F\left(\frac{x}{2}\right)=x^{2 g+1}-2^{2 g} \mathbf{b} x-2^{2 g-1} \mathbf{c} \in \mathcal{O}[x] \subset K[x] .
$$


We have $K\left(\Re_{F}\right)=K\left(\Re_{U}\right), K(\sqrt{\Delta(F)})=K(\sqrt{\Delta(U)})$. Since

$$
\mathbf{S}_{2 g+1}=\operatorname{Perm}\left(\Re_{U}\right)=\operatorname{Gal}(U / K)=\operatorname{Gal}\left(K\left(\Re_{U}\right) / K\right),
$$

we have

$$
\operatorname{Gal}\left(K\left(\Re_{U}\right) / K(\sqrt{\Delta(U)})\right)=\mathbf{A}_{2 g+1} \text {. }
$$

It follows from Remark 6.7 that the Galois extension $K\left(\Re_{U}\right) / K$ is ramified at every prime divisor of 2 (in $K$ ). On the other hand, Lemma 6.3 (a) (applied to $u(x)=U(x)$ ) tells us that the quadratic extension $K(\sqrt{\Delta(U)}) / K$ is unramified at every prime divisor of 2 (in $K)$. Since all the field extensions involved are Galois, $K\left(\Re_{U}\right) / K(\sqrt{\Delta(U)})$ is ramified at every prime divisor of 2 (in $K(\sqrt{\Delta(U)}))$.

Since $K$ is purely imaginary, $K(\sqrt{\Delta(U)})$ is also purely imaginary and therefore (its every field extension, including) $K\left(\mathfrak{R}_{U}\right)$ is unramified at all infinite places (in $K(\sqrt{\Delta(U)}))$.

Remark 6.2 and Lemma 6.3 (a) (applied to $u(x)=U(x)$ ) imply that the field extension $K\left(\Re_{U}\right) / K(\sqrt{\Delta(U)})$ is unramified at all maximal ideals $\mathfrak{b}$ in $\mathcal{O}$ with odd residual characteristic.

\section{Corrigendum to [20]}

- Page 660, the 6th displayed formula: insert $\subset$ between $\operatorname{End}_{\mathrm{Gal}(K)} V_{\ell}(X)$ and End $_{\mathbb{Q}_{\ell}} V_{\ell}(X)$.

- Page 662, Theorem 2.6, line 3: $r_{1}$ should be $r_{2}$.

- Page 664, Remark 2.16: The reference to [23, Theorem 1.5] should be replaced by [23, Theorem 1].

- Page 664, Theorem 2.20: The following additional condition on $\ell$ was inadvertently omitted:

" (iii) If $C$ is the center of $\operatorname{End}(X)$ then $C / \ell C$ is the center of $\operatorname{End}(X) / \ell \operatorname{End}(X)$. ." In addition, "be" on the last line should be "is".

- Page 666, Theorem. 3.3, line 2: $\ell$ should be assumed to be in $P$, i.e. one should read "Then for all but finitely many $\ell \in P \ldots$.. In addition, $X_{n}$ should be $X_{\ell}$ throughout lines 3-6.

- Page 668, Lemma 3.9, line 1: $\operatorname{Isog}_{P}$ should be Is $P$.

- Page 668, Theorem 3.10, line 1: replace $\operatorname{Isog}_{P}\left(\left(X \times X^{t}\right)^{8}, K, 1\right)$ by $\operatorname{Is}_{P}\left(\left(X \times X^{t}\right)^{4}\right.$, $K, 1)$.

- Page 670, Section 5.1, the first displayed formula: $t$ should be $g$.

- Page 672, line 9: $X_{\ell}^{\prime}$ should be $X_{\ell}$.

(The author is grateful to Kestutis Cesnavicius for sending this list of typos.)

Acknowledgments The author is grateful to the referee, whose comments helped to improve the exposition. 


\section{References}

1. Arno, S., Robinson, M.L., Wheeler, F.S.: Imaginary quadratic fields with small odd class number. Acta Arith. 83(4), 295-330 (1998)

2. Cohen, S.D., Movahhedi, A., Salinier, A.: Double transitivity of Galois groups of trinomials. Acta Arith. 82(1), 1-15 (1997)

3. Faddeev, D.K., Sominsky, I.S.: Problems in Higher Algebra, 5th edn. Mir, Moscow (1972)

4. Gao, Sh: Absolute irreducibility of polynomials via Newton polytopes. J. Algebra 237(2), 501-520 (2001)

5. Hall, Ch.: An open-image theorem for a general class of abelian varieties. Bull. Lond. Math. Soc. 43(4), 703-711 (2011)

6. Koch, H.: Number Theory II. Encyclopaedia of Mathematical Sciences, vol. 62. Springer, Berlin (1992)

7. Lang, S.: Algebra, 3rd edn. Addison-Wesley, Reading (1993)

8. Mori, Sh.: The endomorphism rings of some abelian varieties II. Jpn. J. Math. 3(1), 105-109 (1977)

9. Mott, J.L.: Eisenstein-type irreducibility criteria. In: Anderson, D.F., Dobbs, D.E. (eds.) ZeroDimensional Commutative Rings (Knoxville, 1994). Lecture Notes in Pure and Applied Mathematics, vol. 171, pp. 307-329. Marcel Dekker, New York (1995)

10. Mumford, D.: Abelian Varieties. Tata Institute of Fundamental Research Studies in Mathematics, vol. 5, 2nd edn. Oxford University Press, Oxford (1974)

11. Osada, H.: The Galois groups of the polynomials $X^{n}+a X^{l}+b$. J. Number Theory 25(2), 230-238 (1987)

12. Ribet, K.A.: Endomorphisms of semi-stable abelian varieties over number fields. Ann. Math. 101(3), 555-562 (1975)

13. Selmer, E.S.: On the irreducibility of certain trinomials. Math. Scand. 4, 287-302 (1956)

14. Serre, J.-P.: Abelian l-Adic Representations and Elliptic Curves. Advanced Book Classics, vol. 2, 2nd edn. Addison-Wesley, Redwood City (1989)

15. Serre, J.-P.: Topics in Galois Theory. Research Notes in Mathematics, vol. 1. Jones and Bartlett, Boston (1992)

16. Watkins, M.: Class numbers of imaginary quadratic fields. Math. Comp. 73(246), 907-938 (2004)

17. Zarhin, Yu.G.: Hyperelliptic jacobians without complex multiplication. Math. Res. Lett. 7(1), 123-132 (2000)

18. Zarhin, Yu.G.: Very simple 2-adic representations and hyperelliptic jacobians. Mosc. Math. J. 2(2), 403-431 (2002)

19. Zarhin, Yu.G.: Families of absolutely simple hyperelliptic jacobians. Proc. Lond. Math. Soc. 100(1), 24-54 (2010)

20. Zarhin, Yu.G.: Abelian varieties over fields of finite characteristic. Cent. Eur. J. Math. 12(5), 659-674 (2014)

21. Zarhin, Yu.G.: Two-dimensional families of hyperelliptic jacobians with big monodromy. Trans. Amer. Math. Soc. (to appear). arXiv:1310.6532 